\title{
POLÍTICAS PÚBLICAS LOCALES Y DESARROLLO
}

\author{
Braulio Vargas, M. A. \\ COORDINADOR DE LOS PROGRAMAS DE \\ GESTIÓN MUNICIPAL, ESAN \\ bvargas@esan.edu.pe
}

\section{Resumen}

Ofrece una base conceptual sobre las políticas públicas y su extensión hacia políticas de Estado, con énfasis en las políticas de promoción del desarrollo social y económico en el contexto de la descentralización. Reseña la evolución de las políticas públicas, desde el enfoque del Estado benefactor hasta el enfoque moderno del Estado promotor, y luego explica el proceso de diseño y gestión de políticas públicas. El artículo llama la atención sobre las políticas públicas como medio para transformar una situación de insatisfacción social en una situación de satisfacción social.

$\mathbf{E}_{\mathrm{m}}$ presente artículo busca plantear un marco de referencia para concebir políticas públicas locales en el contexto de la descentralización, con particular énfasis en la concepción de políticas de promoción del desarrollo social y económico.

En la primera parte se revisa la evolución reciente de las políticas públicas como herramientas de los gestores públicos: gobierno y administración pública. Se parte del enfoque del Estado benefactor que interviene en los distintos ámbitos de la sociedad, incluyendo el privado, para asistir a la población en sus necesidades insatisfechas, y se continúa con el enfo- que del Estado promotor y eficiente que diseña y gestiona deliberadamente actividades y proyectos destinados a satisfacer las necesidades sociales y a impulsar el potencial de desarrollo local y/o regional, de modo eficiente, con calidad, productividad, transparencia y eficacia en el presente y con proyección hacia el futuro.

En la segunda parte se discute el proceso de concepción y elaboración de políticas públicas a partir de dos grandes componentes: el diseño, que a su vez incluye las fases de diagnóstico y decisión, y la gestión, que a su vez incluye las fases de dirección y difusión. 
De esta manera, el artículo busca consolidar un conjunto de nociones relativamente dispersas para ofrecer al lector una base conceptual sobre las políticas públicas y su extensión hacia la definición de políticas de Estado -políticas que son adoptadas por sucesivas gestiones- y políticas sociales ${ }^{1}$, entendidas ambas como aquellas orientadas, en un primer momento, a asistir y, en un segundo momento, a promover el desarrollo y la satisfacción de las necesidades de la mayor parte de miembros de la sociedad.

\section{El gobierno y las políticas públicas: concepciones}

A la mayoría de personas les resulta cotidiano enterarse o recibir información acerca de las políticas nacionales de carácter económico, social, cultural, entre otros aspectos, en el nivel del gobierno central. Asimismo, están acostumbradas a las políticas de empresa, escuchan con frecuencia frases como «es política de la empresa...», «nuestra política señala que...», «de acuerdo con la política de la empresa...», etc. Pero muy pocas están familiarizadas con el tema de las políticas en los niveles de gobierno local y/o regional. ¿Significa esto que en los gobiernos locales las políticas públicas no existen?

Para aproximarnos a una posible respuesta a esta pregunta, en primer lugar ensayamos un conjunto de razonamientos que nos permitan entender mejor la naturaleza de las políticas públicas, sin por ello

1. Las políticas sociales se diferencian de las políticas de Estado únicamente en que son promovidas, elaboradas o aplicadas por agentes distintos del Estado, como las ONG, instituciones en general o empresas. pretender abarcar todos o la mayoría de aspectos que han jugado un papel clave en su origen.

\subsection{Las políticas públicas desde una concepción tradicional del Estado- Nación}

El desarrollo de la cultura política que impera en países de Occidente está marcado por la construcción de una estructura de poder político-jurídica, que significó una evolución importante con relación a la estructura político-jurídicomilitar, característica de regímenes tribales y posteriormente feudales. El cambio significó dar paso a una estructura eminentemente política conocida como Estado-Nación, transición que empezó hace aproximadamente cuatro siglos y que no se ha detenido.

Esta nueva estructura demostró, en muchos casos, que podía integrar una visión político-demócrata (basada en la libertad de elección) con los intereses y las necesidades sociales. Esta visión política prestaba enorme atención a la dimensión económico-territorial, pues veía en ella la pieza clave para generar una dinámica productiva y comercial que permitiría enfrentar con éxito la problemática social dentro del territorio. Parte del rol del Estado, entonces, fue intervenir en materia económica con el propósito de generar condiciones sine qua non que alentaran una creciente actividad de inventiva, de manufactura y de mercadeo para industrializar el territorio, generar empleo y, por ende, bienestar. En dicho momento se consolidó la imagen del Estado de bienestar y se hicieron patentes los primeros lineamientos o directrices para orientar la intervención y decisiones del Estado en materia política, social y económica. 
Estas intervenciones y decisiones son entendidas como políticas nacionales, las que bajo una estructura centralizada de gobierno y gestión pública se operativizan a través de instancias de carácter vertical, como es el caso de los ministerios. Intuitivamente se puede decir que en un contexto de descentralización se requiere de un proceso por el cual surjan políticas públicas también desde los niveles de gobierno subnacional, pero a través de instancias de carácter horizontal, como pueden ser las redes público-privadas o la especialización (en forma de gerencias) al interior de los gobiernos regionales y locales.

Asimismo, debe notarse que en un mundo influenciado por procesos de mundialización, internacionalización y globalización, los enfoques tradicionales y centralistas de las políticas públicas resultan muy inefectivos. Como contrapeso a los procesos exógenos, debe propenderse a un enfoque de localización que se operativice a través de la descentralización de las políticas públicas.

\subsection{Las políticas públicas: supuestos para el cambio en un contexto de descentralización}

El enfoque expuesto permite una primera definición de políticas públicas. Según Dye (1998), son los proyectos gubernamentales y las actividades administrativas propuestas y gestionadas desde el Estado (a través de un gobierno y de una administración pública) con la finalidad de satisfacer las necesidades de una sociedad en el presente y también en el futuro. Como este autor señala: «Todo aquello que los gobiernos deciden hacer o no hacer».
Como correlato de lo anterior, en el centro de la discusión sobre políticas públicas (de índole económica, jurídica, cultural, etc.) deberían encontrarse las denominadas políticas sociales de carácter multidimensional, e inclusive de marcado énfasis en aspectos económico-productivos, ambientales e institucionales. Esto significa que las políticas públicas no deben estar segmentadas como tiende a suceder bajo un enfoque sectorial de gobierno y administración pública. Por el contrario, en un contexto de descentralización, las políticas públicas apuntan a distintas dimensiones que afectan el mayor o menor bienestar y desarrollo, dimensiones que deben necesariamente retroalimentarse entre sí.

No obstante, la historia de países en desarrollo, como el Perú, demuestra que de alguna manera se ha contaminado la esencia original de las políticas públicas, lo que aparentemente indica que han existido distorsiones serias a nivel del gobierno y de la administración pública.

En primer lugar, los gobiernos de turno (dimensión político-estratégica) no han logrado demostrar capacidad para generar confianza en su facultad de integrar intereses y expectativas de los diferentes actores, tanto sociales como empresariales, ni mucho menos para inspirar compromisos colectivos. Tampoco han estado ajenos a actos de corrupción y/o a manejos poco transparentes de los recursos públicos o a las tentaciones populistas que han hecho de las políticas o, por ejemplo, de los programas sociales, un vehículo de clientelismo político. En el caso concreto del Perú, las consecuencias se han sentido en sus diversas manifestaciones, desde golpes de Estado encabezados por militares, pasando por regíme- 
nes democráticos nada participativos, hasta regímenes democráticos más participativos, pero con gran déficit de gobernabilidad democrática.

En segundo lugar, la situación de la administración pública (dimensión ejecutiva o técnica) no ha sido más alentadora. Por un lado, su desempeño ha mostrado pocos resultados y casi ninguna voluntad para brindar calidad de servicio a los usuarios de servicios públicos, es decir, la sociedad en su conjunto. La administración pública se convierte, por momentos, en una dimensión en exceso burocrática que, lejos de atender las necesidades de los ciudadanos y de los agentes productivos, genera barreras al buen clima necesario para facilitar los negocios y los contratos sociales (la mano visible del desarrollo). Esta aparente inacción que suele aquejarla se ve reforzada a lo largo del tiempo por la relativamente alta movilidad de gestores públicos emprendedores -quienes no encuentran incentivos para permanecer en el sector público- y la complacencia de quienes permanecen en la administración pública. Bajo estas circunstancias, la administración pública en general difícilmente puede esperar retener, ni mucho menos captar, profesionales motivados e innovadores que transformen la cultura e innoven las prácticas de gestión para revitalizar tanto las políticas como la administración pública.

Por otro lado, la administración pública ha estado sujeta a la voluntad política del gobierno de turno y a los intereses de grupos dominantes al interior de las instituciones públicas. La mayor parte de la evidencia recolectada a lo largo de las dos últimas décadas señala algunas de estas malas prácticas de gestión, como las siguientes: a) Las empresas públicas y las agencias del Estado (ministerios y entes especializados) han sido fuente predilecta de empleo de partidarios que no necesariamente cuentan con los conocimientos y preparación que un determinado puesto requiere.

b) Al contrario de lo que ocurre en la administración privada, se han designado más personas de las necesarias para un mismo tipo de función, lo que ha contribuido a que los procesos y procedimientos hayan desbordado sus tamaños y tiempos óptimos. Algunos trámites que deben realizar ciudadanos o empresas enfrentan un sinnúmero de firmas, visados, pasos previos, traslados entre distintas oficinas (incluso entre distintas instituciones), verificaciones y pagos de diversa índole que, en muchos casos, no guardan relación directa con el propósito del trámite original y sólo alimentan mayores burocracias.

c) Los fondos públicos han tenido, en instancias lejanas a los mecanismos de control del gobierno central, un uso muy distinto al planificado, lo que ha restado recursos para atender otros fines de mayor prioridad, como la salud, la seguridad alimentaria, la familia, la educación, el desarrollo económico local, etc. En estos casos, los ciudadanos no logran razonablemente entender cómo las instituciones de la administración pública pueden operar si han perdido su real foco de atención, que consiste en servir a las necesidades de la sociedad. Además, con frecuencia estas necesidades son atendidas como si se estuviera haciendo favores y no cumpliendo una función. 
d) Retomando el enfoque tradicional de Estado-Nación, encontramos que resulta muy difícil diseñar y gestionar políticas públicas efectivas. Como se expuso anteriormente, ni el gobierno ni la administración pública tienen las capacidades, la inspiración, la legitimidad ni la confianza suficientes para diseñar y gestionar políticas. Esto alimenta la evidente necesidad de impulsar urgentes procesos de modernización y reforma del Estado, no sólo a nivel de la administración pública, como normalmente se sostiene, sino también a nivel del gobierno y de nuestra cultura política.

En consecuencia, es razonable pensar que para los niveles de gobierno regional y de gobierno local existen pocos o ningún referente en el tema del diseño y gestión de políticas públicas. Esto es una suerte de «rumor de barrio»: todos hablan de las políticas públicas, pero pocos saben a ciencia cierta qué son, para qué sirven, cómo se diseñan y qué consideraciones intervienen en su elaboración, implementación, administración, control y ajuste, entre otros aspectos.

\subsection{Las políticas públicas desde una concepción moderna del Estado}

El punto anterior mostró un panorama con pocas perspectivas para el tema de las políticas públicas locales. No obstante, conviene analizar ciertos fenómenos convergentes que han empezado a generar oportunidades para el diseño y gestión de este tipo de políticas.

El primero de ellos es la transformación progresiva del Estado-Nación, que ha dejado de ser un Estado de bienestar o benefactor para convertirse en un Estado promotor y moderno cada vez más conectado al ciudadano, en el sentido más estricto de la palabra. Esta nueva figura se basa en un enfoque de racionalidad económica que responde al avance industrial y del capitalismo hacia una economía social de mercado. Las evidencias más claras refieren que este avance se inicia a partir de la segunda mitad del siglo XX en un entorno caracterizado por economías industriales en reconstrucción (luego de las guerras mundiales), movimientos obreros, reivindicación de la mujer, emergencia del sistema socialista y oposición al capitalismo, etc.; elementos que determinaron un hito histórico en el papel asumido por el Estado en cuanto a intervenir en todos los ámbitos de actuación de la sociedad en beneficio de la ciudadanía (democracia representativa).

En esta transición aún deben resolverse fricciones y vacíos en cuanto a, por ejemplo, marcos regulatorios y desarrollo económico local como contrapeso a la mayor exposición y niveles de competencia derivados de la mayor apertura del mercado. Por competencia no solamente entendemos la pugna entre empresas -y sus respectivos productos- de diferentes localidades por ganar cuota de mercado, sino también la competencia de economías (locales y regionales) por recursos estratégicos (como el agua), capitales de inversión y posiciones de poder político y/o comercial que, entre otros y en su conjunto, hacen la diferencia entre la polarización y la equidad en la distribución de oportunidades, riqueza y bienestar.

El segundo fenómeno es la emergencia de la participación de los ciudadanos, en especial de los actores locales, en los procesos políticos y de toma de decisiones en materia de desarrollo. Esto viene 
ocurriendo de forma paralela a los procesos de descentralización y regionalización que, para ser viables, requieren exaltar la participación y el compromiso de todos a fin de enfrentar las restricciones y las oportunidades del colectivo.

En este contexto, la democracia y los derechos de los ciudadanos se van perfeccionando y surge la preocupación por incrementar las competencias del ámbito local para afrontar la participación ciudadana en las decisiones públicas. Nuevamente, se hace evidente que hablar de políticas públicas no se restringe a tomar decisiones en un solo sentido o aspecto, sino que obliga a entender las relaciones causa-efecto con otras áreas o dimensiones. De ahí el carácter multidimensional de las políticas públicas.

Combinando ambos razonamientos, se hace necesario utilizar un enfoque híbrido que articule el fortalecimiento del Estado con la construcción de ciudadanía. Este enfoque privilegia la figura de un Estado moderno y promotor que evoluciona más allá del enfoque asistencialista o paternalista -por el cual las necesidades de la sociedad son definidas por el Estado- para adoptar un enfoque basado en la promoción del desarrollo integral, que beneficie tanto a ciudadanos como a empresas y otros agentes interesados en el desarrollo local, regional y/o nacional.

Precisamente este matiz integrador requiere que los gestores públicos y privados no pierdan de vista factores elementales de carácter nacional, suprarregional y global (por ejemplo, internacionalización de la economía, formación de bloques económicos), porque, como es sabido, la realidad local se construye también a partir de las tendencias e innovaciones presentes incluso en sociedades muy distantes, y la globalización permite una gran movilidad de informaciones, tendencias, modas y recursos que irremediablemente serán, en todo o en parte, asimilados por nuestra propia sociedad.

Esta perspectiva moderna de las políticas públicas en un escenario de estados modernos que se descentralizan y construyen competencias en los ámbitos locales de gobierno - a fin de entrar en contacto directo con el ciudadano y con la globalización- alude directamente a que el gobierno y la gestión pública descentralizados precisan de compartimentos territoriales en los cuales se pueda delegar competencias y funciones de gestión y ejecución en temas públicos, y de promoción en temas privados.

En este sentido los gobiernos locales son políticamente relevantes, y ello presupone que su rol no debe ser solamente el de concentrar todos sus esfuerzos en tareas administrativas y de prestación de servicios, sino también el de diseñar políticas públicas, emplear más eficientemente los recursos públicos, priorizar las actividades y proyectos de mayor impacto en el bienestar y el desarrollo (costo-efectividad) y que tiendan a facilitar la intervención de las fuerzas de mercado a favor del impulso a la economía local. Este y otros aspectos vienen progresivamente emergiendo, máxime cuando se cae en cuenta de que en un escenario de descentralización los gobiernos locales se ven reforzados al tomar el poder que concentraban los estados centralistas.

Una escena diferente a la descrita supone que los gobiernos locales quedarían sometidos a las directrices emanadas desde el nivel del gobierno nacional, que 
centraliza las decisiones y los recursos. Esto implica un alto costo de oportunidad derivado de preferir optar por el centralismo en lugar de aprovechar el potencial y las ventajas que presentan los niveles de gobierno subnacionales.

Finalmente, es prudente reiterar que los procesos descritos no son ajenos a la corriente expansiva de la globalización a escala mundial, corriente que impulsa el desarrollo y uso de nuevas tecnologías, la movilidad del capital, la comunicación y la información acerca de los logros científicos y culturales, entre otras ventajosas posibilidades para unos, que representan amenazas e inequidades para otros. En efecto, la globalización ha dejado al descubierto que existe una gran desigualdad entre economías territoriales, y que las más perjudicadas son las que no cuentan con las condiciones para competir a escala global ni para beneficiarse de los flujos de capital o de los desarrollos científicos originados en fuerzas de mercado que ya no dependen en su integridad de países, sino, más bien, de economías ${ }^{2}$. En este contexto, las políticas públicas son un elemento urgente para reaccionar ante las influencias del entorno.

Esta reflexión es muy importante, toda vez que la concentración del progreso en manos de un poder económico dominante opaca la presencia del Estado, incluso en aquellas decisiones que se consideran

2. El término economía es preferentemente empleado en determinados contextos de carácter supranacional: la Unión Europea, el Foro de Cooperación Asia-Pacífico e incluso ciudades y corporaciones multinacionales que concentran capitales, conocimientos, procesos, tecnologías y/o servicios específicos en los que se han especializado, como Hong Kong (zona autónoma), Miami o Seattle. materia pública. Esto sucede principalmente en economías de menor desarrollo relativo. Lo opuesto ocurre en economías de mayor desarrollo relativo, donde el Estado está fuertemente identificado con los agentes económicos hospedados en su ámbito territorial; por ende, sus decisiones y políticas están deliberadamente orientadas a concentrar los beneficios de la globalización en involuntario desmedro de las economías menos influyentes.

Como evidencia de lo anterior podemos analizar el caso de compañías o proyectos asentados en territorios ricos en recursos naturales. Ocurre frecuentemente que dichos proyectos o compañías se esfuerzan en explotar las fuentes de ventaja comparativa local (recursos naturales o mano de obra barata, por lo general) para alimentar su propia competitividad, pero sin mayor efecto sobre la competitividad del territorio ni de las personas afectadas (positiva o negativamente) por dicha actividad económica. En este caso, la compañía es competitiva, pero el espacio local no es competitivo. Sucede que la competitividad territorial se ha desplazado desde la zona de explotación hacia la zona donde la empresa o el proyecto conducen la rentabilidad, el aprendizaje y los dividendos de sus operaciones; vale decir, al espacio local donde se toman las decisiones, se produce o sistematiza el conocimiento y/o se pagan los impuestos.

Lo anterior nos invita, en el caso concreto de países en desarrollo como el Perú, a reflexionar en torno a dos preguntas; una de naturaleza estratégica y otra de carácter operativo:

- ¿Qué tipo de intervenciones e iniciativas deben impulsarse desde el Estado, en sus distintos niveles de gobierno, 
para enfrentar fenómenos globales de rápida concentración de riqueza y bienestar, polarización de la pobreza (con regímenes de semiesclavitud), males endémicos (como el sida) y merma irreversible del espacio natural y de las condiciones esenciales para la supervivencia (agua, capa de ozono, bosques)?

- Para elaborar una plataforma favorable a las iniciativas referidas en la pregunta anterior y en razón de que la difícil tarea de generar bienestar y desarrollo exige de la cooperación de todos, ¿qué condiciones deben generarse, mantenerse y reforzarse a fin de armonizar los intereses y expectativas de los actores presentes en el ámbito local/nacional con la oferta política del gobierno de turno y con los procesos de democratización?

En el fondo, ambas preguntas se refieren a políticas públicas que den continuidad a la relación entre los gobiernos, la administración pública y los actores presentes en la comunidad en sentido doble: mediante políticas que ayuden a impulsar iniciativas de desarrollo desde el nivel del gobierno central hacia los niveles regionales y locales (con el fin de sintonizar intereses y expectativas e inspirar el trabajo colectivo) y, a la inversa, mediante políticas que surjan desde los gobiernos locales y regionales para impulsar la competitividad territorial y la integración con otros territorios. De este modo será posible incorporar las dinámicas locales (de carácter multidimensional) a una dinámica más integradora que siempre deberá existir (de carácter nacional). En otras palabras: pensar globalmente y actuar desde una perspectiva de desarrollo local.

\section{Diseño y gestión de políticas públicas}

La primera parte de este artículo estuvo dedicada a compartir dos ideas centrales sobre la razón de ser de las políticas públicas. La primera idea se basa en la figura del Estado benefactor centralista que con una lógica keynesiana decide impulsar iniciativas (programas, proyectos, etc.) a través de las cuales busca intervenir en distintos ejes temáticos e influir sobre los agentes del mercado para propiciar un clima favorable al desarrollo y bienestar de la sociedad.

La segunda idea se proyecta hacia la figura de un Estado moderno, descentralizado y flexible que en virtud de sus mayores competencias requiere perfeccionar los mecanismos por los cuales identifica, prioriza, planifica, organiza y gestiona sus intervenciones con el propósito mixto de proveer servicios públicos y de promover el desarrollo integral en el corto, mediano y largo plazo; es decir, con un enfoque institucionalizado e independiente del estilo de gestión y del sistema de toma de decisiones que adopten los sucesivos gobiernos.

\subsection{Definición de políticas públicas}

A diferencias de sus pares en el ámbito de la gestión empresarial o privada, las políticas en el sector público se denominan, valga la redundancia, públicas en tanto en ellas convergen dos factores: a) el Estado, como sujeto principal y b) la sociedad, como objeto principal. Este enunciado significa un aporte mayúsculo al entendimiento de las políticas públicas, pues permite intuir que no todo lo estatal es necesariamente público, ni que todo lo social es público. Luego, las políticas 
públicas se ubican en la intersección de lo estatal con lo social (Emilio Craglia, 2004).

Desde la perspectiva de sujeto principal, los influyentes estudios de Luis Aguilar (2000) proponen una noción descriptiva de las políticas públicas con base en el aspecto institucional que, en síntesis, parte del supuesto de que si existe una cierta institucionalidad, entonces la política consiste en: a) el diseño de una acción colectiva intencional, b) el curso que efectivamente toma la acción como resultado de las muchas decisiones e interacciones que comporta y, en consecuencia, c) los hechos reales que la acción colectiva produce.

Esta definición es distinta de la definición descriptiva inicialmente propuesta -según la cual las políticas son proyectos y actividades que un Estado diseña y gestiona a través de un gobierno y una administración pública-, porque va más allá de la mera acción para proponer que las políticas surgen como producto de procesos de gestión (decisiones e interacciones) cuyo diseño e implementación deben conducir a lograr y/o mantener unos resultados predefinidos.

Según el razonamiento anterior, es evidente que las políticas públicas coinciden con las políticas empresariales en cuanto a su finalidad: cursos de acción que obedecen a decisiones colectivas, pensados e implementados para mejorar las posibilidades (en cierta forma garantizar) de lograr los resultados deseados.

Esta definición más elaborada de las políticas públicas llama la atención sobre tres aspectos: Primero, se trata de comportamientos deliberados más que de re- acciones casuales. Segundo, no todas las actividades, leyes o proyectos del gobierno o de la administración pública constituyen políticas. Tercero, no se debe confundir estrategias con políticas, pues las primeras son modos planificados para llegar a un objetivo, mientras las segundas constituyen decisiones y comportamientos que buscan favorecer que las estrategias se ejecuten como se han planeado.

Finalmente, dado que las políticas públicas tienen como sujeto al Estado y como objeto a la sociedad, son políticas estatales o materia de Estado. Por ende, compete al Estado diseñar y gestionar dichas políticas incluso cuando los servicios públicos han sido privatizados o concesionados al sector privado, toda vez que lo predominante sigue siendo el carácter público y no el sentido empresarial de la gestión de dichos servicios.

\subsection{Gestión de políticas públicas}

Uno de los vicios comunes en materia de políticas públicas en países en desarrollo como el Perú es pretender definir como política de Estado (o política de empresa en el caso del sector privado) todas las iniciativas y proyectos de un determinado gobierno. Esto es errado porque, como hemos visto, además de la acción se requiere de la decisión deliberada y de la vigilancia para, en cierta medida, garantizar que el curso de acción tomado conduzca a los resultados esperados. No obstante, se tiende a mantener esta idea equivocada, de modo que el próximo gobierno interpretará que debe generar sus propias políticas, pues parte del supuesto de la superioridad de sus propuestas o políticas frente a las del gobierno anterior. 
En realidad, dado su carácter intencional y/o deliberado, las políticas deben proyectarse al futuro, a fin de que los procesos de involucramiento, gestión y manifestación de impactos muestren sus resultados, independientemente de la bondad o debilidad de las estrategias, acciones y proyectos que se planteen. Esto significa que una verdadera política es tal si y sólo si los sucesivos gobiernos la adoptan y continúan gestando. Cuando esto sucede, se dice que la política pública es una política de Estado, toda vez que el Estado trasciende a la duración de los gobiernos de turno, e incluso a la de las administraciones públicas.

Según el razonamiento expuesto, las políticas públicas se convierten en otro de los recursos escasos y valiosos que deben ser gestionados con eficacia y eficiencia. En el caso concreto de los gobiernos locales, se puede afirmar, por ejemplo, que el programa del Vaso de Leche tiene las características de una política de Estado, porque es continuado por gobiernos que no lo diseñaron. No obstante, falta verificar si, como se ha definido, se consiguen los resultados esperados, ya que se han diseñado cursos de acción encaminados a dicho propósito. Es decir, es necesario conocer con certeza si el Vaso de Leche efectivamente satisface una necesidad social para los genuinos beneficiarios. Intuitivamente se puede percibir que este programa social no reúne las características necesarias para ser considerado una política de Estado.

En suma, las políticas públicas solamente son aquellas actividades, proyectos y/o decisiones deliberadas (no quiere decir que toda iniciativa o decisión es una política pública) que buscan satisfacer necesidades sociales, aun cuando en al- gunos casos no lleguen a hacerlo. Deben tenerse en cuenta las diversas razones por las cuales las necesidades sociales no terminan de ser satisfechas: porque la población continuará creciendo (sobre todo la más vulnerable), porque ningún esfuerzo público contará con el presupuesto suficiente para resolver una situación muy deficitaria (pensar lo contrario es, más bien, ilusorio) o porque un enfoque asistencialista que perdure de modo indefinido no es sostenible.

\subsection{Aplicación de políticas públicas}

Llegamos a una definición final: cualquiera sea el motivo que se persigue con una política pública, el centro de atención siempre deberá estar determinado por el propósito o fin último de satisfacer necesidades sociales. Hacemos la salvedad de que con esto no nos estamos refiriendo a necesidades insatisfechas de un tipo de individuo o población objetivo (lo que se consigue con la asistencia directa), sino, más bien, a las necesidades de todos los individuos de la sociedad en el presente y en el futuro.

Por ejemplo, una política en materia económica deberá diseñarse de modo tal que coadyuve a alcanzar fines sociales, como la atención de salud, la educación primaria, el saneamiento ambiental, la seguridad alimentaria, la producción y mercadeo de los productos agrícolas provenientes de comunidades campesinas especializadas en la agricultura, el control de la natalidad, la medicina preventiva, la orientación familiar sobre paternidad responsable, la atención materno-infantil, la promoción de la micro y pequeña empresa, el acceso de la mujer a mayores y mejores oportunidades de incorporación a la economía del hogar, entre otros. 
Al repasar estos temas, el lector muy posiblemente habrá caído en la cuenta del grado de relevancia de las políticas públicas y, más específicamente, de las políticas sociales. Si se conciben equivocadamente es razonable pensar que la incidencia de las acciones y proyectos gubernamentales será, en el mejor de los casos, limitada. No hace falta mencionar que lo mismo sucederá si las políticas públicas se diseñan, gestionan y controlan también de manera errada.

Surge entonces la pregunta acerca de los elementos que dan soporte a un acertado proceso de definición, diseño, gestión y evaluación de políticas. Contrariamente a lo que pudiera pensarse, esto no dependerá exclusivamente de una mente iluminada que realice un excelente trabajo de gabinete, sino, más bien, de que se preste la máxima atención a dos factores clave:

\section{a) La participación de la sociedad}

En tiempos recientes se ha acumulado suficiente evidencia acerca de que el gobierno perderá legitimidad si no cuenta con el apoyo ciudadano a su gestión. Si ello sucede, en lugar de contar con la participación activa de los ciudadanos en las decisiones y acciones de desarrollo, se tendrá en ellos una fuente poderosa de oposición. En estas condiciones, incluso las políticas más elaboradas difícilmente contribuirán a satisfacer necesidades sociales.

Es conveniente aclarar que la participación no se consigue porque una ley lo dictamine, sino porque se generen condiciones y canales de contacto entre los ciudadanos y el gobierno. En este sentido, la educación cívica será una política prioritaria de todo gobierno, independientemen- te de su estilo de gestión y del tipo de oferta política que busque implementar. Si es así, será más probable que la ciudadanía se involucre en los procesos de desarrollo.

\section{b) La gestión gubernamental}

La gestión gubernamental debe ser transparente, de calidad, eficaz (lograr metas de atención) y eficiente. Esta combinación es como un imán que atrae la atención del ciudadano y de los actores locales acerca de la calidad de la administración pública bajo el gobierno de turno. Esto es clave porque transmite el mensaje de un mejor trabajo a un costo que no es mayor y cuyos resultados incrementan el nivel de satisfacción de los contribuyentes (y ciudadanos en general), quienes percibirán el eficiente uso de los tributos que pagan con el dinero que tanto esfuerzo les ha costado ganar.

\subsection{El proceso de elaboración de políticas públicas}

Partiendo del razonamiento de que las políticas obedecen al propósito de satisfacer necesidades sociales (situación esperada o resultado), entonces deberán existir situaciones de insatisfacción social de orden relativo (insumo). Se dice de orden relativo porque mientras para una sociedad sus actuales estándares de educación podrían no ser satisfactorios, para otra sociedad esos mismos estándares podrían ser más de lo que esperaba alcanzar. Luego, la elaboración de políticas públicas busca transformar una situación de insatisfacción social en otra de satisfacción social, proceso en el cual no faltarán fases o momentos intermedios. Estas fases han sido objeto de estudio y se ha llegado a dos importantes aproximaciones: 
La primera aproximación corresponde a la de las «fases primarias», trabajadas en profundidad por Graglia. Las siguientes fases son las principales:

a) Diagnóstico y decisión: es el diseño de políticas desde un enfoque técnicopolítico.

b) Dirección y difusión: es la gestión de políticas desde un enfoque políticoadministrativo.

Jones (1984) y Meny y Thoening (1992) han planteado una aproximación de naturaleza secuencial que comprende: identificar la situación insatisfactoria, formular un conjunto de soluciones, tomar una decisión, llevar la decisión a la acción y monitorear y evaluar los resultados. En realidad esta aproximación concuerda muy bien con la clasificación de Graglia.

a) Diagnóstico. Busca detectar y jerarquizar necesidades insatisfechas y problemas (insumos) y luego desarrollar alternativas de solución.

b) Decisión. Las alternativas posibles (insumos) son valoradas de manera comprensiva. Como resultado de ello se obtienen proyectos concretos, con sus respectivos objetivos y tareas.

c) Dirección. El énfasis es la gestión de alta productividad. Los proyectos y actividades priorizados (insumos) deben tener su correlato en actividades dirigidas que deben ejecutarse (implementación y operación) y evaluarse. La evaluación es parte de la gestión y comprende el análisis de las actividades ejecutadas y su ajuste o corrección en función de parámetros o indicadores de control. El resultado son las actividades ajustadas. d) Difusión. Las actividades dirigidas (insumos) que materializan los proyectos provenientes de la etapa de dirección basan su éxito no sólo en la gestión eficiente, sino también en la difusión, análisis y corrección de las actividades proyectadas y ejecutadas.

Por un lado, la difusión debe realizarse en la etapa inmediata anterior y durante la implementación de las actividades proyectadas y debe cubrir de manera amplia tanto el interior de la institución pública (a fin de inspirar la adhesión) como el exterior (para que sus beneficiarios, los ciudadanos, entiendan su objetivo e implicancias y se sientan motivados a participar). Por otro lado, la difusión debe extenderse más allá de la etapa de ejecución (implementación y operación) para incluir la evaluación de las actividades previamente comunicadas y, de ser necesario, su corrección. Como resultado de esta etapa, las actividades son comunicadas de manera transparente (decisiones y acciones).

Al revisar las cuatro fases del proceso de elaboración de las políticas se observa que el input o insumo del diseño de políticas públicas es el diagnóstico, mientras su resultado o output es la decisión. Por tanto, diseñar políticas supone traducir necesidades insatisfechas y problemas en proyectos que apuntan a la satisfacción de dichas necesidades y a la solución de problemas. Así, el input de la gestión es el insumo o input de la dirección, mientras su output es la difusión. En consecuencia, gestionar políticas públicas supone convertir proyectos cuya ejecución ha sido decidida en actividades que han de difundirse (socializar e implementar). 


\section{Referencias bibliográficas}

AGUILAR VILLANUEVA, Luis F. 2000. Estudio Introductorio. En:- (ed.). La hechura de las políticas. $2^{\mathrm{a}}$ ed. México: Miguel Ángel Porrúa. Vol. 2, págs. 15-84.

ALLISON, Graham T. 2000. Modelos conceptuales y la crisis de los misiles cubanos. En Aguilar Villanueva, Luis F. (ed.). La hechura de las políticas. $2^{\mathrm{a}}$ ed. México: Miguel Ángel Porrúa. Vol. 2, págs. 119-200.

DYE, Thomas R. 1998. Understanding public policy. Upper Saddle River, New Jersey: Prentice Hall.

GRAGLIA, Emilio. 2004. Gobernar y administrar por políticas públicas. Córdoba, Argentina: Universidad Católica de Córdoba.

GRINDLE, Merilee S. y THOMAS, John W. 1991. Public choices and policy change: The political economy of reform in developing countries. Baltimore: The John Hopkins University Press.

JONES, Charles O. 1984. An introduction to the study of public policy. $3^{\mathrm{a}} \mathrm{ed}$. Monterrey: Brooks/Cole.
KLIKSBERG, Bernardo. 1998. Seis tesis no convencionales sobre participación. Instituciones y Desarrollo. N. ${ }^{\circ}$ 2, págs. 131169.

LOWI, Theodore. 1964. American business, public policy, case studies and political theory. World Politics. N. ${ }^{\circ} 16$, págs. $677-$ 687.

MENY, Yves y THOENIG, Jean-Claude. 1992. Las políticas públicas. Barcelona: Ariel.

SALAZAR VARGAS, Carlos. 1999. Las políticas públicas. $2^{\mathrm{a}}$ ed. Pontificia Universidad Javeriana.

SUBIRATS, Joan. 1996. Democracia: participación y eficiencia. Gestión y Análisis de Políticas Públicas. N. ${ }^{\circ}$ 5-6, págs. 35-44.

WILDAVSKY, Aaron. 1980. The self-evaluating organization. En: Nachmias, David (ed.). The practice of policy evaluation. New York: St. Martin's Press. Págs. 441460. 